\title{
Complete Blood Count Alterations of COVID-19 Patients in Riyadh, Kingdom of Saudi Arabia
}

\author{
Abozer Y. Elderdery ${ }^{(D)}{ }^{1}$ Ahmed M. E. Elkhalifa $\left(\mathbb{D},{ }^{2,3}\right.$ Abdullah Alsrhani $\left(\mathbb{D},{ }^{1}\right.$ \\ Khalid I. Zawbaee $\mathbb{D}^{4}{ }^{4}$ Saad M. Alsurayea ${ }^{D},{ }^{5}$ Faisal K. Escandarani ${ }^{(D)}{ }^{5}$ \\ Abdulaziz H. Alhamidi $\mathbb{D}^{6}{ }^{6}$ Hadeil M. E. Idris $\mathbb{D}^{7},^{7}$ Anass M. Abbas $\mathbb{D}^{1},{ }^{1}$ Manar G. Shalabi $\mathbb{D}^{1}{ }^{1}$ \\ and Jeremy Mills ${ }^{8}{ }^{8}$

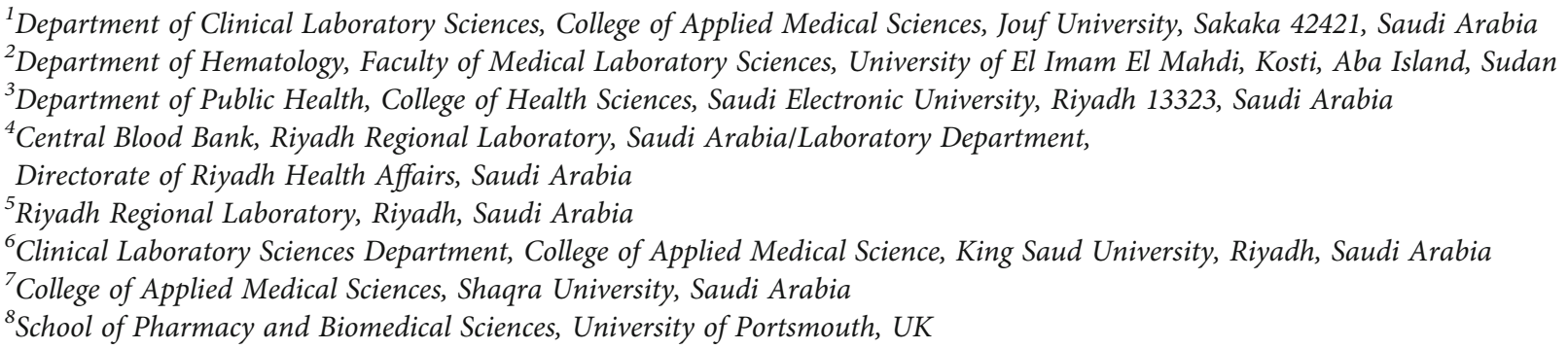

Correspondence should be addressed to Abozer Y. Elderdery; ayelderdery@ju.edu.sa and Ahmed M. E. Elkhalifa; ahmedelnour2003@yahoo.com

Received 31 October 2021; Accepted 24 December 2021; Published 31 January 2022

Academic Editor: Palanivel Velmurugan

Copyright (C) 2022 Abozer Y. Elderdery et al. This is an open access article distributed under the Creative Commons Attribution License, which permits unrestricted use, distribution, and reproduction in any medium, provided the original work is properly cited.

\begin{abstract}
This study is aimed at evaluating the association between Coronavirus disease 19 (COVID-19) and the primary complete blood count (CBC) parameters in confirmed positive patients. In a retrospective cross-sectional study, 384 files of patients with confirmed COVID-19 diagnosis hospitalized at King Saud Medical City, Riyadh, were chosen randomly as a study group for hematological parameters, with another 384 non-COVID-19 files of patients without history of any disease which could influence the hematological profile were selected as a control group. The gender, age, and nationality of the control group were matched with those of the study group. Anemia and thrombocytopenia prevalence was significantly higher in COVID-19 positive patients compared with negative. However, the positive cases were 3.4 times more likely to be anemic and approximately 5.3 times as likely to be thrombocytopenic, while the prevalence of leukopenia showed no statistical significance between the two groups. However, the Mean Cell Volume (MCV), Total White Blood Cell (WBC) count, lymphocyte count, and basophil count median values showed a nonsignificant difference between the two groups. Anemia and thrombocytopenia may be highly developed in severe positive cases, and therefore, further studies are recommended to validate these findings.
\end{abstract}

\section{Introduction}

Over the last two decades, 3 new coronavirus subtypes have emerged, in outbreaks that provoke great concern at a global level. These are sequentially SARS-COV, MERS-CoV, and SARS-CoV-2, respectively, the Severe Acute Respiratory Syndrome Coronavirus, Middle-East Respiratory Syndrome
Coronavirus, and Severe Acute Respiratory Syndrome Coronavirus-2 [1]. In manifestation, these exhibit broad similarities, including a shared tendency of progression to severe symptomatology, associated with high incidence of both morbidity and mortality. Having said this, comparing their clinical presentations and complications distinguishes clear variability of respective processes [2]. 
The contagious COVID-19 novel coronavirus is rapidly evolving and spreading throughout the world, significantly affecting millions of people worldwide [3]. The disease is caused by the zoonotic positive-strand RNA virus known as SARSCoV-2 (Respiratory Syndrome Coronavirus 2) [4]. The disease has caused severe socioeconomic and health disruption $[5,6]$. Symptomatic infected patients with COVID-19 usually present with mild clinical symptoms including but not limited to fever, dry cough, and alteration in taste and/or smell sensation, where they usually recover in a few days $[7,8]$. However, patients with severe symptoms, especially those with comorbidities such as chronic diseases, may quickly progress to develop pneumonia and acute respiratory distress syndrome days after the onset of the disease, with an increased mortality rate $[8,9]$, suggesting that COVID-19 causes a multisystem disorder [10]. Since the initial reports considering COVID-19 as only pneumonia, accumulated data have revealed that intravascular coagulation and coagulopathy are common in severely infected patients, causing the rise in mortality rate $[11,12]$. These data suggested that the coagulation pathway is very active in COVID-19-infected individuals $[10,13]$.

Interestingly, in several hematological laboratory investigations into lymphocytes, peripheral eosinophils and neutrophils were altered significantly in COVID-19 patients, thus being suggested as a potential indicator for both disease progression and effectiveness of therapy [14]. Importantly, some studies also suggest an association between COVID19 infection and reduction in hemoglobin $(\mathrm{Hb})$ levels [15, 16]. This suggested association had been previously reported with other types of pneumonia [17]. Therefore, we conducted a retrospective analysis to evaluate the association between COVID-19 and the main blood parameters including the $\mathrm{Hb}$ concentration, other red blood cell (RBC) indices, TWBCs, and platelet indices among COVID-19 hospitalized cases in Saudi Arabia compared with nonpositive cases.

\section{Materials and Methods}

2.1. Study Design, Setting, and Population. The investigation is retrospective, hospital-based, and cross-sectional, conducted at the King Saud Medical City, Riyadh Regional Laboratory Centre, KSA, from October to November 2020 to compare $\mathrm{Hb}$ concentration, $\mathrm{RBC}$ counts, RBC indices, WBCs, and platelet indices among confirmed positive hospitalized cases and negative subjects.

2.2. Sample Size and Sampling. Using EPI info software at a confidence level of 95\%, 384 anonymous COVID-19 patients' files were selected randomly from electronic medical records and clinical laboratory databases, recruited as a study group for $\mathrm{Hb}, \mathrm{RBC}$ counts, and red cell indices. The corresponding 384 files for COVID-19-negative patients matched for age, gender, and nationality without a history of blood diseases were chosen randomly as a control group. The COVID-19 diagnosis was confirmed by a positive Reverse-Transcriptase-Polymerase-Chain-Reaction (RTPCR) SARS-CoV-2 assay result [18, 19]. The samples for the COVID-19 investigation were obtained using nasopharyngeal swab samples.
2.3. Ethical Approval. The Saudi Electronic University Institutional Review Board (SEU-IRB) issued ethical approval according to the Helsinki Declaration principles after the research protocol was reviewed before data collection began. The ethical approval number was SEUREC-CHS20113-REC.

2.4. Inclusion and Exclusion Criteria. Hospitalized positive cases and negative cases were identified and then excluded if they had a history of any disease, which could influence the hematological profile, were under treatment with drugs affecting complete blood count (CBC), and had cardiovascular disease or liver disease.

2.5. Statistical Analysis. The laboratory's findings, plus clinical and personal data extracted from the patient's files, were entered into the Statistical Package for the Social Sciences (SPSS Software version-25; IBM Corp., Armonk, NY, USA), then revised, and coded. Continuous data were expressed and presented as mean standard deviation $( \pm S D)$ with the data distributed normally according to the significance of the test of normality. Median interquartile range (IQR) was applied for the nonnormally distributed data. Continuous variables were compared for the normally distributed data using independent Student's $t$-test and for nonnormally distributed data using the Mann-Whitney $U$ test. A $p$ value $\leq$ 0.05 was selected as the threshold of significance.

\section{Results}

3.1. General Characteristics of the Patients (Table 1). The gender profile of COVID-19 patients and the control group in this study showed 221 patients $(57.6 \%)$ were males and $163(42.4 \%)$ were females. The median age was $49.0 \pm$ 23.75 for the COVID-19 patient group and $45.0 \pm 26.0$ for the non-COVID-19 patients (range 19-98 years) with a nonsignificant difference regarding age $(p=0.165)$. The study and control group's nationalities included citizens from KSA $(173,45.1 \%)$ and non-Saudi $(210,54.9)$.

3.2. Laboratory Findings. Table 2 represents the comparison of $\mathrm{Hb}$ concentration, $\mathrm{RBC}$ counts, and red cell indices between COVID-19 patients and the non-COVID-19 control group. Our findings stated that $\mathrm{Hb}$ concentration, $\mathrm{RBC}$ count, haematocrit (Hct), mean cell hemoglobin $(\mathrm{MCH})$, and mean cell hemoglobin concentration (MCHC) median values are significantly decreased in COVID-19 patients compared with the control group $(p \leq 0.01, p \leq 0.01, p=$ 0.041 , and $p \leq 0.01$, respectively). The median values of the red cell distribution width (RDW) show a statistically significant increase in COVID-19 patients compared with the control group $(p \leq 0.01)$. However, the MCV median values showed no significant difference between the two groups $(p=0.264)$.

Table 3 explains a significant decrease in the eosinophil count and platelet count median values among COVID-19 patients compared with controls $(p \leq 0.01$ and $p \leq 0.01$, respectively).

The median values of Mean Platelet Volume (MPV) showed a statistically significant increase among COVID19 patients compared with the control group $(p \leq 0.01)$. 
TABLE 1: Distribution of age, gender, and nationality of the COVID-19 patients and the control group.

\begin{tabular}{|c|c|c|c|c|}
\hline Variables & Category & $\begin{array}{l}\text { COVID-19 patients } \\
\text { Frequency } N(\%)\end{array}$ & $\begin{array}{c}\text { Non-COVID-19 patients } \\
\text { Frequency } N(\%)\end{array}$ & $P$-value \\
\hline \multirow{3}{*}{ Gender } & Male & $221(57.6)$ & $221(57.6)$ & \\
\hline & Female & $163(42.4)$ & $163(42.4)$ & \\
\hline & Total & $384(100)$ & $384(100)$ & \\
\hline \multirow{2}{*}{ Age } & $18-98$ & $384(100)$ & $384(100)$ & \\
\hline & Median $\pm \mathrm{IQR}$ & $49.00 \pm 23.75$ & $45.0 \pm 26.0$ & 0.165 \\
\hline \multicolumn{5}{|l|}{ Nationality } \\
\hline \multirow{2}{*}{ Saudi } & Male $N(\%)$ & $71(18.5)$ & $116(30.2)$ & \\
\hline & Female $N(\%)$ & $101(26.3)$ & $113(29.4)$ & \\
\hline \multirow{2}{*}{ None Saudi } & Male $N(\%)$ & $151(39.3)$ & $104(27.1)$ & \\
\hline & Female $N(\%)$ & $61(15.9)$ & $51(13.3)$ & \\
\hline Total & & 384 & 384 & \\
\hline
\end{tabular}

IQR: interquartile range.

TABle 2: Comparison of hemoglobin concentration, RBC counts, and red cell indices of the COVID-19 patient and the non-COVID-19 patient control group.

\begin{tabular}{lccc}
\hline \multirow{2}{*}{ Parameter } & $\begin{array}{c}\text { COVID-19 patients } \\
n=384\end{array}$ & $\begin{array}{c}\text { Non-COVID-19 patients } \\
n=384\end{array}$ & $p$ value \\
& Median IQR & $12.30 \pm 2.40$ & $\leq 0.01^{*}$ \\
\hline $\mathrm{Hb}(\mathrm{g} / \mathrm{dL})$ & $11.00 \pm 3.50$ & $37.80 \pm 7.28$ & $\leq 0.01^{*}$ \\
PCV (L/L) & $34.40 \pm 9.35$ & $4.49 \pm 0.89$ & $\leq 0.01^{*}$ \\
RBCs $\left(\times 101^{2} / \mathrm{L}\right)$ & $4.20 \pm 1.30$ & $85.7 \pm 58.00$ & $=0.264$ \\
MCV (fL) & $85.60 \pm 9.30$ & $27.90 \pm 3.20$ & $\leq 0.041^{*}$ \\
MCH (pg) & $27.60 \pm 3.70$ & $32.50 \pm 1.70$ & $\leq 0.01^{*}$ \\
MCHC (\%) & $32.20 \pm 1.90$ & $14.90 \pm 2.60$ & $\leq 0.01^{*}$ \\
RDW (\%) & $15.30 \pm 3.78$ & &
\end{tabular}

${ }^{*}$ Significance at the level $\leq 0.05 . N$ : number; IQR: interquartile range.

TABLE 3: WBC and platelet indices of the COVID-19 patient and the non-COVID-19 patient control group.

\begin{tabular}{|c|c|c|c|}
\hline Parameter & $\begin{array}{c}\text { COVID-19 patients } \\
n=384 \\
\text { Median IQR } \\
\end{array}$ & $\begin{array}{c}\text { Non-COVID-19 patients } \\
n=384 \\
\text { Median IQR } \\
\end{array}$ & $p$ value \\
\hline Total WBC count $\left(\times 10^{9} / \mathrm{L}\right)$ & $7.79 \pm 4.34$ & $7.95 \pm 4.38$ & 0.983 \\
\hline Neutrophil (\%) & $62.4 \pm 21.4$ & $61.2 \pm 25.42$ & 0.173 \\
\hline Lymphocyte (\%) & $24.5 \pm 18.3$ & $24.65 \pm 22.0$ & 0.604 \\
\hline Monocyte (\%) & $8.1 \pm 3.7$ & $8.1 \pm 3.97$ & 0.591 \\
\hline Eosinophil (\%) & $1.6 \pm 2.7$ & $2.15 \pm 3.08$ & $\leq 0.01^{*}$ \\
\hline Basophil (\%) & $0.5 \pm 0.5$ & $0.6 \pm 0.5$ & 0.124 \\
\hline Mean Platelet Volume (MPV) & $9.3 \pm 1.8$ & $9.00 \pm 1.8$ & $\leq 0.01^{*}$ \\
\hline Platelet count $\left(\times 10^{9} / \mathrm{L}\right)$ & $249.0 \pm 128.0$ & $305.00 \pm 161.0$ & $\leq 0.01^{*}$ \\
\hline
\end{tabular}

${ }^{*}$ Significance at the level $\leq$ 0.05. N: number; IQR: interquartile range; WBC: White Blood Cell.

However, the Total White Blood Cell count, lymphocyte count, and basophil count median values showed no significant difference between the two groups.
According to WHO classification regarding anemia, leucopenia, and thrombocytopenia [20, 21], Table 4 illustrates the comparison of hematological cytopenias between 
TABLE 4: Hematological cytopenia among the COVID-19 patients and non-COVID-19 patients.

\begin{tabular}{|c|c|c|c|c|}
\hline Hematological abnormality & $\begin{array}{c}\text { COVID-19 patients }(N=384) \\
n(\%)\end{array}$ & $\begin{array}{c}\text { Non-COVID-19 patients }(N=384) \\
n(\%)\end{array}$ & OR $(95 \%$ CI $)$ & $P$ value \\
\hline Anemia & $275(62.8)$ & $163(37.2)$ & $3.4(2.53-4.61)$ & $\leq 0.01$ \\
\hline Leukocytopenia & $17(48.6)$ & $18(51.4)$ & $0.94(0.47-1.85)$ & $=0.86$ \\
\hline Thrombocytopenia & $52(82.5)$ & $11(17.5)$ & $5.3(2.72-10.34)$ & $\leq 0.01$ \\
\hline
\end{tabular}

COVID-19 patients and non-COVID-19 patients. The prevalence of anemia among COVID-19 patients was significantly higher compared to non-COVID-19 patients $(62.8 \%$ vs. $37.2 \%$, respectively). However, the COVID-19 patients were 3.4 times more likely to be anemic. The prevalence of thrombocytopenia was also significantly higher in COVID19 patients compared to non-COVID-19 patients $(82.5 \%$ vs. $17.5 \%$, respectively). COVID-19 patients were approximately 5.3 times to be thrombocytopenic. The prevalence of leukopenia showed a nonstatistical significance between the two groups ( $48.6 \%$ vs. $51.4 \%$, respectively).

\section{Discussion}

In this study, a majority of subjects $(221,57.6 \%)$ were male with a median age of $49.0 \pm 23.75$. This finding is consistent with other studies reporting similar results; $52.1 \%$ of the patients were males with a median age of 47 in a study by Usul and coworkers [22]. Liang et al. [18] found that about $56 \%$ of all cases were males and their median age was 59 . Additionally, a study by Chen et al. [19] reported a median age of 41 and $56 \%$ of the patients being males. In another study, $72 \%$ were males and the mean age was $61.67 \pm 15.60$ . Usul et al. [22] stated that $69.3 \%$ were males and the average age was $46.2 \pm 15.5$ years. This finding could be explained by the fact that males are more vulnerable to infection with COVID-19 than females due to biological variation in the immune system and genetic factors, as reported by epidemiological studies [23, 24]. Additionally, lifestyle also contributes to the higher infectivity in males, for example, smoking, drinking alcohol, and less commitment to social distancing [18]. Besides that, females were acting more responsibly toward the COVID-19 crisis than males as reported by Bwire and coworkers [23]. However, other authors reported an equal male to female ratio [25].

We found that $\mathrm{Hb}$ concentration, RBC count, HCT, $\mathrm{MCH}$, and $\mathrm{MCHC}$ median values were all significantly decreased in COVID-19 subjects compared with controls. This finding agrees with Yuan and coworkers [26], who found that severe and critically ill patients had significantly decreased $\mathrm{RBC}$ and $\mathrm{Hb}$ and with another study reporting a rapid decline of $\mathrm{Hb}$ and $\mathrm{RBC}$ among COVID-19 patients [27].

Additionally, a study by Mei et al. [28] found that the red blood cell parameters (RBC, Hb, and HCT) were significantly reduced in patients where COVID-19 was severe. The exact mechanism of how COVID-19 causes anemia is not fully understood but suggested to be via inhibition of erythropoiesis in the bone marrow. On the contrary, Usul et al. [22] stated that $\mathrm{Hb}$ levels in COVID-19-positive patients were found to be significantly higher than those in COVID-19-negative patients. This discrepancy from our results could be attributed to differences in study population characteristics such as the presence of underlying chronic diseases and cigarette smoking, which could directly affect the RBC profile. Those factors were not the exclusion criteria as mentioned by the authors [22, 29]. Fan and his colleagues [30] revealed that on admission to the hospital, most COVID-19 patients had a normal CBC (normal Hb, WBC, and platelet count).

In this study, the median values of RDW show a statistically significant increase among COVID-19 subjects against the control group. This finding is consistent with that of Lee and coworkers [31] that nearly half (49.7\%) of the patients hospitalized for COVID-19 were found to have elevated RDW values at presentation and with Wang et al., who found that the morphological parameters (RDW-CV and RDW-SD) were significantly higher in the severe COVID19 group [32].

The current study found no significant difference in Total White Blood Cell count, lymphocyte count, and basophil count median values between the two groups. However, several studies reported lymphocytopenia as the most common finding among the COVID-19 cases. Meanwhile, other studies observed neutrophilia [30, 33-35]. A decrease in lymphocyte count is strongly associated with the severity of the disease. This may explain the conflict in results between the current study and the mentioned reports, as most of them collected the data from severely ill or Intensive Care Unit (ICU) cases, reflecting poor prognosis of the disease. The present study found a significant decrease in the eosinophil count and platelet count median values among COVID-19 patients compared with controls. This finding is in agreement with previous reports [25, 33-36]. Thrombocytopenia is common among COVID-19 patients through inhibition of thrombopoiesis, immunological destruction of platelets, and consumption due to lung injury [37].

\section{Conclusion}

COVID-19 patients may have complications of anemia such as hypoxia and coronary and pulmonary insufficiency because of low $\mathrm{Hb}$ concentration. Bleeding disorders may also occur among COVID-19 patients because of thrombocytopenia. Therefore, transfusion of packed RBCs and platelets may assist in alleviating and avoiding complications like anemia and bleeding, in turn reducing the mortality rate from COVID-19 infection. 


\section{Data Availability}

The data used to support the findings of this study are included in the article. Should further data or information be required, these are available from the corresponding author upon request.

\section{Conflicts of Interest}

The authors declare that there is no conflict of interest regarding the publication of this paper.

\section{Acknowledgments}

We would like to thank the Deanship of Scientific Research and Postgraduate Studies, Jouf University, Sakaka, Saudi Arabia, for the financial support (DSR-2021-01-035). We would also like to thank the staff of King Saud Medical City Lab, Riyadh.

\section{References}

[1] J. Guarner, "Three emerging coronaviruses in two Decades," American Journal of Clinical Pathology, vol. 153, no. 4, pp. 420-421, 2020.

[2] J. Gerges Harb, H. A. Noureldine, G. Chedid et al., "SARS, MERS and COVID-19: clinical manifestations and organsystem complications: a mini review," Pathogens and Disease, vol. 78, no. 4, 2020.

[3] M. Cascella, M. Rajnik, A. Aleem, S. Dulebohn, and R. Di Napoli, "Features, evaluation, and treatment of coronavirus (COVID-19)," StatPearls, vol. 35, no. 5, pp. 20-28, 2021.

[4] A. Sharma, S. Tiwari, M. K. Deb, and J. L. Marty, "Severe acute respiratory syndrome coronavirus-2 (SARS-CoV-2): a global pandemic and treatment strategies," International Journal of Antimicrobial Agents, vol. 56, no. 2, 2020.

[5] T. Singhal, "A review of coronavirus disease-2019 (COVID19)," Indian Journal of Pediatrics, vol. 87, no. 4, pp. 281-286, 2020.

[6] A. Alsrhani, K. Junaid, S. Younas, S. S. M. Hamam, and H. Ejaz, "COVID-19 pandemic: through the lens of science, a painstaking review," Clinical Laboratory, vol. 66, no. 10/2020, 2020.

[7] A. Thakar, S. Panda, P. Sakthivel et al., "Chloroquine nasal drops in asymptomatic \& mild COVID-19: an exploratory randomized clinical trial," The Indian Journal of Medical Research, vol. 153, no. 1, p. 151, 2021.

[8] H. Ejaz, A. Alsrhani, A. Zafar et al., "COVID-19 and comorbidities: deleterious impact on infected patients," Journal of Infection and Public Health, vol. 13, no. 12, pp. 1833-1839, 2020.

[9] C. M. Roberts, M. Levi, M. McKee, R. Schilling, W. S. Lim, and M. P. W. Grocott, "COVID-19: a complex multisystem disorder," British Journal of Anaesthesia, vol. 125, no. 3, pp. 238242, 2020.

[10] M. Z. Zuo, Y. G. Huang, W. H. Ma et al., "Expert recommendations for tracheal intubation in critically ill patients with noval coronavirus disease 2019," Chinese Medical Sciences Journal, vol. 35, no. 2, 2020.
[11] M. Levi and T. Iba, "COVID-19 coagulopathy: is it disseminated intravascular coagulation?," Internal and Emergency Medicine, vol. 16, no. 2, pp. 309-312, 2021.

[12] H. Asakura and H. Ogawa, "COVID-19-associated coagulopathy and disseminated intravascular coagulation," International Journal of Hematology, vol. 113, no. 1, pp. 45-57, 2021.

[13] P. Zamboni, "COVID-19 as a vascular disease: lesson learned from imaging and blood biomarkers," Diagnostics, vol. 10, no. $7,2020$.

[14] C. Huang, Y. Wang, X. Li et al., "Clinical features of patients infected with 2019 novel coronavirus in Wuhan, China," The Lancet, vol. 395, no. 10223, pp. 497-506, 2020.

[15] G. Lippi and C. Mattiuzzi, "Hemoglobin value may be decreased in patients with severe coronavirus disease 2019," Hematology, Transfusion and Cell Therapy, vol. 42, no. 2, pp. 116-117, 2020.

[16] M. C. Reade, L. Weissfeld, D. C. Angus, J. A. Kellum, and E. B. Milbrandt, "The prevalence of anemia and its association with 90-day mortality in hospitalized community-acquired pneumonia," BMC Pulmonary Medicine, vol. 10, no. 1, 2010.

[17] N. Rahimi-Levene, M. Koren-Michowitz, R. Zeidenstein, V. Peer, A. Golik, and T. Ziv-Baran, "Lower hemoglobin transfusion trigger is associated with higher mortality in patients hospitalized with pneumonia," Medicine, vol. 97, no. 12, 2018.

[18] C. Liang, J. Cao, Z. Liu et al., "Positive RT-PCR test results after consecutively negative results in patients with COVID-19," Infectious Diseases, vol. 52, no. 7, pp. 517-519, 2020.

[19] Z. H. Chen, Y. J. Li, X. J. Wang et al., "Chest CT of COVID-19 in patients with a negative first RT-PCR test," Medicine, vol. 99 , no. $26,2020$.

[20] E. Shevlin and R. A. Morrow, "Comparative performance of the Uni-Gold ${ }^{\mathrm{TM}} \mathrm{HSV}-2$ Rapid: A point-of-care HSV-2 diagnostic test in unselected sera from a reference laboratory," Journal of Clinical Virology, vol. 61, no. 3, pp. 378-381, 2014.

[21] J. Gerard, F. Dubois-Galopin, M. Gardembas-Pain et al., "Refractory anaemia with ringed sideroblasts (RARS) associated with marked thrombocytosis: a provisional entity in the WHO classification of haematological malignancies," Annales de Biologie Clinique, vol. 63, no. 6, pp. 653-659, 2019.

[22] E. Usul, I. San, B. Bekgoz, and A. Sahin, "Role of hematological parameters in COVID-19 patients in the emergency room," Biomarkers in Medicine, vol. 14, no. 13, pp. 1207-1215, 2020.

[23] G. M. Bwire, "Coronavirus: Why men are more vulnerable to COVID-19 than women?," SN Comprehensive Clinical Medicine, vol. 2, no. 7, pp. 874-876, 2020.

[24] R. Zhong, L. Chen, Q. Zhang et al., "Which factors, smoking, drinking alcohol, betel quid chewing, or underlying diseases, are more likely to influence the severity of COVID-19?," Frontiers in Physiology, vol. 11, article 623498, 2021.

[25] J. M. Jin, P. Bai, W. He et al., "Gender differences in patients with COVID-19: focus on severity and mortality," Front Public Health, vol. 8, no. 2, p. 152, 2020.

[26] X. Yuan, W. Huang, B. Ye et al., "Changes of hematological and immunological parameters in COVID-19 patients," International Journal of Hematology, vol. 112, no. 4, pp. 553-559, 2020.

[27] A. Berzuini, C. Bianco, A. C. Migliorini, M. Maggioni, L. Valenti, and D. Prati, "Red blood cell morphology in patients with COVID-19-related anaemia," Blood Transfusion, vol. 19, no. 1, pp. 34-36, 2021. 
[28] Y. Mei, S. E. Weinberg, L. Zhao et al., "Risk stratification of hospitalized COVID-19 patients through comparative studies of laboratory results with influenza," EClinicalMedicine, vol. 26, 2020.

[29] M. Rossato and A. Di Vincenzo, "Cigarette smoking and COVID-19,” Pulmonology, vol. 27, no. 3, pp. 277-278, 2021.

[30] B. E. Fan, "Hematologic parameters in patients withCOVID19 infection: a reply," American Journal of Hematology, vol. 95, no. 8, 2020.

[31] J. J. Lee, S. M. Montazerin, A. Jamil et al., "Association between red blood cell distribution width and mortality and severity among patients with COVID-19: a systematic review and meta-analysis," Journal of Medical Virology, vol. 93, no. 4, pp. 2513-2522, 2021.

[32] C. Wang, R. Deng, L. Gou et al., "Preliminary study to identify severe from moderate cases of COVID-19 using combined hematology parameters," Annals of Translational Medicine, vol. 8, no. 9, p. 593, 2020.

[33] N. Chen, M. Zhou, X. Dong et al., "Epidemiological and clinical characteristics of 99 cases of 2019 novel coronavirus pneumonia in Wuhan, China: a descriptive study," The Lancet, vol. 395, no. 10223, pp. 507-513, 2020.

[34] F. A. Naoum, A. L. Z. Ruiz, F. H. O. Martin, T. H. G. Brito, V. Hassem, and M. G. L. Oliveira, "Diagnostic and prognostic utility of WBC counts and cell population data in patients with COVID-19," International Journal of Laboratory Hematology, vol. 43, no. S1, pp. 124-128, 2021.

[35] O. Pozdnyakova, N. T. Connell, E. M. Battinelli, J. M. Connors, G. Fell, and A. S. Kim, "Clinical significance of CBC and WBC morphology in the diagnosis and clinical course of COVID-19 infection," American Journal of Clinical Pathology, vol. 155, no. 3, pp. 364-375, 2021.

[36] J. Xie, Z. Tong, X. Guan, B. Du, and H. Qiu, "Clinical characteristics of patients who died of coronavirus disease 2019 in China," JAMA Network Open, vol. 3, no. 4, 2020.

[37] P. Xu, Q. Zhou, and J. Xu, "Mechanism of thrombocytopenia in COVID-19 patients," Annals of Hematology, vol. 99, no. 6, pp. 1205-1208, 2020. 